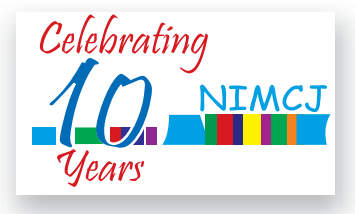

${ }^{1}$ Dr. A S M Anwarul Kabir Registrar

Surgery Dept.

Holy Family Red Crescent Medical

College (HFRCMC), Dhaka

${ }^{2}$ Farjana Akhter

Senior Staff Nurse

National Institute of Cardiovascular

Diseases (NICVD), Dhaka

${ }^{3}$ Dr. Mahbuba Sharmin

Medical Officer

Bangabandhu Sheikh Mujib

Medical University (BSMMU)

${ }^{4}$ Dr. Khaleda Akhter

Registrar

${ }^{5}$ Mosammat Beauty Begum

Senior Staff nurse

National institute of Chest

Diseases and Hospital, Dhaka

${ }^{6}$ Dr.Arup Kumar Saha

Associate professor and Head

Dept. of Dental Public Health

City Dental College \& Hospital,

Dhaka

7 Prof. Dr. Imtiaz Ahmed

Professor, Surgery Dept.

4, 7

Holy Family Red Crescent Medical College and Hospital, Dhaka

Correspondence

Dr. A S M Anwarul Kabir

Registrar, Surgery Dept.

Holy Family Red Crescent Medical

College and Hospital

e-mail : dr.anwar28@gmail.com

\title{
Knowledge, Attitude and Practice of Staff nurses on Hospital Acquired Infections in tertiary care Hospital of Dhaka city
}

\author{
A S M A Kabir ${ }^{1}$, F Akhter ${ }^{2}$, M Sharmin ${ }^{3}$, K Akhter ${ }^{4}$, M B Begum ${ }^{5}$, \\ A K Saha ${ }^{6}$, I Ahmed ${ }^{7}$
}

\begin{abstract}
Introduction : Hospital acquired Infections (HAls) are called those infections that were not present at the time of patient' hospitalization in a hospital and have been acquired after hospitalization. Nurses are an important part of the any healthcare team who play a unique role in the control of Hospital acquired infections.

Objective : The aim of this study was to investigate the level of knowledge, attitudes, and practices of staff nurses about preventing the spread of hospital acquired infections (HAls) at tertiary care Hospital of Dhaka city.

Methods and Materials : This descriptive cross-sectional study was done among nurses having two years experience from two tertiary level hospitals in Dhaka city during January to June 2017. Self administered questionnaire containing different set of questions regarding knowledge, attitude and practice on HAI were used as a tool for data collection. Questionnaire was supplied to all staff nurses available at different in-patient wards of these two hospitals. Only 234 staff nurses who completed and returned the questionnaire were included in this study. Data were analyzed using Microsoft excel 2013 software.
\end{abstract}

Result : Staff nurses were found to have good knowledge, moderately positive attitude but poor practice in prevention of hospital acquired infections. About $95 \%$ of the participants considered that prevention of HAls were a valuable part of their role. About $65 \%$ of the staff nurses had received formal training regarding hand hygiene. The $100 \%$ of participants felt that they would be less likely to transmit infection to the patients if they performed hand-hygiene. About $64 \%$ of them argued that hand hygiene agents were not readily available in current settings. Regarding practice, only $6 \%$ performed hand hygiene before patients contact and $27 \%$ of the staff nurses reported that they often forgot to perform hand hygiene.

Conclusion : The finding of this study revealed a good knowledge of infection prevention among the majority of participants with relatively minimal level of practice. For strengthening the knowledge, attitude and practice towards HAls, there is in need of developing regular training program and monitoring on performance feedback regarding hand hygiene is recommended.

Key Words : HAls. KAP. Hand-hygiene. Hand-washing.

Northern International Medical College Journal Vol. 10 No. 1 July 2018, Page 347-350

\section{Introduction}

Hospital Acquired Infections (HAIs) are not present at the time of patient' hospitalization but acquired after admission in to hospital. It is also referred to as Nosocomial infections. The time frame definition of HAIs is at least 48-72 hours after hospitalization, 3 days after leaving the hospital, 30 days after surgery or 1 year after the implant. Hospital acquired infections (HAIs) are a common global challenge mainly in low and middle-income countries. ${ }^{1}$ An estimated $10 \%$ of hospitalized patients in developed countries and $25 \%$ in developing countries develop HAIs and subsequently results in adverse healthcare outcomes as increased hospital stay, economic burden, significant morbidity, and mortality. ${ }^{1}$

The high burden of HAIs is due to lack of standardized infection prevention program which was neglected due to limited resources, poor sanitary conditions and hygiene practices. ${ }^{2}$ In 2010, a data report for HAIs cases among 2,473 hospitals showed an increased number of infections associated with the use of medical equipment, most of which were displayed in surgical units. Also, other researchers reported 
that patients who underwent surgical procedures had a greater chance of developing HAIs, compared to other patients. ${ }^{3}$ HAIs associated morbidity and mortality are preventable through infection prevention strategy like, proper hand hygiene. ${ }^{4} \mathrm{~A}$ prevalence survey conducted under the auspices of WHO in 55 hospitals of 14 countries representing 4 WHO Regions (Europe, Eastern Mediterranean, South-East Asia and Western Pacific) showed an average of $8.7 \%$ of hospital patients had HAIs. At any time, over 1.4 million people worldwide suffer from infectious complications acquired in hospital. ${ }^{5}$

HAIs are mostly transmitted by healthcare workers (HCW) who fail to practice infection prevention measures. Hence, Healthcare workers including nurses, doctors are front line of protecting themselves and patients from infection. ${ }^{3}$ Identifying existing infection control knowledge attitudes, and practices (KAP) among health care workers (HCWs) is a key first step in developing a successful infection control program. In an effort to raise awareness and provide guidance in combating HAIs in resource limited settings (RLS), the World Health Organization (WHO) launched the Global Patient Safety Challenge: Clean Care is Safer Care campaign. A cornerstone of the program is to decrease HAIs through improving hand hygiene among healthcare workers. ${ }^{6,7}$ Most HAIs are transmitted by health care personnel who fail to practice proper hand washing procedures or change gloves between patient contacts. Health care workers such as nurses, doctors can be a major source of pathogens. ${ }^{8}$

As members of the health care team, nurses play a very important role in HAI control. Nurses must have sufficient information and necessary skills in this field. The results of a study conducted by Darawad et al. on nursing students in Yemen showed that most nursing students have low levels of knowledge, a positive attitude, and a moderate practice about infection control. The education, training, motivation regarding HAIs has a positive impact on retention of KAP in all categories of health workers including nurses to prevent infections that was evidenced in previous researches. ${ }^{9}$ Therefore, this study aimed to investigate the knowledge, attitude and practice on hospital acquired infection prevention among health care workers (staff nurses) at tertiary care hospital.

\section{Materials And Methods}

This descriptive-cross-sectional study was done among staff nurses in two tertiary care Hospitals, Dhaka Medical College Hospital and Holy Family Red Crescent medical College hospital of Dhaka city during January-June, 2017.

Following inclusion and exclusion criteria a questionnaire was supplied to all staff nurses available at different in-patient wards of these two hospitals. Among 234 nurses who responded, filled completely and returned the questionnaire were included in this study.
Inclusion criteria: Only staff nurses who completed at least diploma in nursing or having higher degree, 2 years experience in nursing service and agreed to participate in this study.

Exclusion criteria: Staff nurses having less than 2 years working experience and were not to agree to participate and staff nurses who did not complete and returned data sheet were excluded from this study sample. Data was analyzed using Microsoft excel 2013 software. Permission from Ethical review board of these two hospitals was obtained and written consent was taken from all participants in this study.

Instruments and Techniques: Structured, self administered questionnaire prepared following the guide line of "WHO hand hygiene knowledge questionnaire for health care workers". It was consisting of questions on knowledge, attitude and practices on HAIs. Knowledge was assessed using 10 questions with options either "yes" or "no". Attitude and practice were assessed using a set of 14 and 8 questions respectively. The participants were given "yes" or "no" options to select based on their attitude and practice regarding HAIs. All the questions were subjected to a pre-testing prior to the study and obtained suggestions were taken into consideration. A scoring system was used where 1 point was awarded for each correct response to knowledge, positive attitudes, and good practices. Incorrect knowledge, negative attitudes, and poor practices were given 0 points. A score greater than $75 \%$ was considered as good, $50 \%$ $-74 \%$ moderate and less than $50 \%$ poor.

Knowledge-is clear awareness and understanding on infection prevention activities when caring patients.

Attitude-is s personal view on infection prevention activities when caring patients.

Practice-is a skill on infection prevention activities when caring patients.

\section{Results}

Highest proportion of the participants( 95\%) were aware of the fact that washing hands with soap or an alcohol based antiseptic decreases the risk of transmission of hospital acquired pathogens. Similarly, (91\%) of them disagreed to the fact that hand washing is not necessary if the hand are not visibly dirty. A larger proportion (79\%) of the participants still considered that gloves provided complete protection against acquiring/ transmitting infections. Only (88\%) of them realized that same pair of gloves cannot be worn for multiple patients as long as there is no visible contamination on the gloves and it should be changed after each patient contact. The percentage of participants who considered that healthcare-associated pathogens can be found on normal, intact patient skin was $(78 \%)$ whereas the rest (12\%) believed that intact skin does not harbor pathogens. The knowledge that when using alcohol 
based antiseptics, hands should be rubbed until dry prevailed among $70 \%$ of the participants. The fact that hand hygiene act should be performed before and after direct patient contact was agreed upon by $91 \%$ of the study population. About $77.3 \%$ of the participants had good knowledge regarding HAIs. However, $22.7 \%$ still lacked good knowledge regarding HAIs

Table 1: Knowledge on Hospital acquired infections (correct responses):

No Questions regarding Knowledge no of participant $(\mathrm{N}=234) \%$

K1 Gloves provide complete

$\begin{array}{lll}\text { protection against acquiring/transmitting } & 49 & 21\end{array}$

infection (false)

K2 Healthcare-associated pathogens can be

found on normal, intact patient skin (true)

183

78

K3 Washing your hands with soap or an alcohol based antiseptic decreases the risk transmissionof hospital acquired pathogens (true)

K4 If my hands are not visibly dirty, there is no need to wash my hands prior to patient contact (false)

213

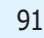

K5 Use of an alcohol based antiseptic for hand

hygiene is as effective as soap and water

if hands are not visibly dirty (true)

K6 Gloves should be worn if blood or body

fluid exposure is anticipated (true)

K7 When using alcohol based antiseptics, I should keep rubbing my hands until dry (true)

K8 There is no need to wash hands before doing procedures that do not involve bodily fluids (false) 218

K9 Hand hygiene should be performed before and after direct patient contact (true)

K10 I can wear the same pair of gloves for

multiple patients as long as there is no

visible contamination on the gloves (false)

In relation to attitude of the staff nurses, $(65 \%)$ of the staff nurses had received formal training regarding hand hygiene and (57\%) thought their supervisors stressed on the importance of hand hygiene. $(27 \%)$ of the staff nurses reported that they often forgot to perform hand hygiene. Majority of the staff nurses $100 \%$ felt that they would be less likely to transmit infection to the patient if they performed hand-hygiene. Regarding various hindrances in adoption of hand hygiene 64\% participants identified that hand hygiene agents (alcohol based hand sanitizer or soap and water) were not always available. $84 \%$ identified that clean towels to dry their hands after washing were not always available. $56 \%$ identified that the sinks were inconveniently located. $95 \%$ participants considered that prevention of HAIs is a valuable part of nurse's role. Regarding attitude on HAIs $64 \%$ showed moderate attitude.
Table 2: Attitude on Hospital acquired infections (Responses on agreement)

No Questions regarding attitude no of participant $(\mathrm{N}=234) \%$

A1 Hand Hygiene agents are not always available $149 \quad 64$

A2 Clean towels to dry my hands after

washing are not always available $\quad 196 \quad 84$

A3 Gloves are always available when needed $\quad 134 \quad 57$

A4 Sinks are inconveniently located $\quad 134 \quad 56$

A5 Sinks are not available $\quad 79 \quad 34$

A6 Hand hygiene agents cause Irritation and dryness $58 \quad 25$

A7 Hand hygiene interferes with

HCW-patient interactions $\quad 116 \quad 50$

$\begin{array}{llll}\text { A8 I often forget to perform hand hygiene } & 64 & 27\end{array}$

A9 I have a very low risk of acquiring infections from my patients. $\quad 78$

A10 If I perform hand hygiene, I am less likely to transmit infections to my patients. $234 \quad 100$

A11 Prevention of HAIs is a valuable part of HCWs role 223

A12 I have received training about the importance of hand hygiene 152

A13 The importance of hand hygiene is emphasized by my clinical supervisors.

A14 I would feel uncomfortable reminding a HCW to perform hand hygiene.

98

With respect to hand hygiene practices, $79 \%$ of the participants had performed hand hygiene after going to toilet. Only $43 \%$ of the participants performed hand hygiene before caring for wound and $61 \%$ after caring for the wound. Only $6 \%$ performed hand hygiene before patient contact and $29 \%$ performed hand hygiene act after patient contact. If the hands felt or looked dirty, $71 \%$ of the participants performed hand hygiene. Practice of hand hygiene after removal of gloves was performed by $37 \%$ of the participants. Practice was poor only $36 \%$ practiced on HAI prevention.

Table 3: Practice on Hospital acquired infections (Always/often responses) No Questions regarding practice no of participant $(\mathbf{N}=234) \quad \%$ of hand wash

P1 Before Patient Contact $\quad 14 \quad 6$

$\begin{array}{lll}\text { P2 After Patient Contact } & 67 & 29\end{array}$

P3 If they look or feel hand is dirty $167 \quad 71$

P4 After going to the toile $\quad 185 \quad 79$

P5 After contact with blood or bodily fluids $\quad 198 \quad 85$

P6 Before caring for a wound $\quad 101 \quad 43$

P7 After caring for a wound $\quad 142 \quad 61$

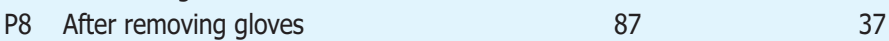

\section{Discussion}

In order to reduce HAIs in any hospital, knowledge of HAIs and compliance to methods in preventing them like proper practice of aseptic and antiseptic precautions by health care workers are very much essential. This study was done to assess the level of knowledge, attitudes, and practices of staff nurses working in tertiary hospitals.

In our study among the staff nurses, we found good knowledge 
among the staff nurses on HAIs and measures to prevent it. This was consistent with the study conducted among the HCWs in Ethiopia and Nepal in similar settings. ${ }^{10,11}$ Our study revealed that still $35 \%$ did not received any formal training in hand hygiene. A study conducted in India regarding the knowledge, attitude and practice of different group of HCWs about infection control concluded that training has a positive impact on the improvement of KAP in health care personnel. They also suggested that development of continuous training program for all HCWs is necessaryy. ${ }^{12}$

More than half (64\%)of the participants reported that hand hygiene agents like- alcohol based hand sanitizer, soap and water were not easily available. Similarly, $(84 \%)$ reported, clean towels to dry the hands after washing were also not always available. The sinks for washing purposes were inconveniently located and inadequate at the hospital-settings. These findings are in accordance to a similar study conducted in Srilanka ${ }^{13}$. Thus increasing the supplies necessary for hand washing and institutional support is essential in combating the substandard practices in hand hygiene. ${ }^{9,11,12}$ Limited accessibility of hand hygiene facilities has been shown to be an important risk factor for poor adherence to recommendations. ${ }^{13}$ Our study showed that although there were a number of limitations in currenthealth care settings, staff nurses had moderately positive attitude towards limiting hospital acquired infections control.

More than half $(57 \%)$ of the participants reported that the importance of hand hygiene was emphasized by their clinical supervisors. An important finding from this study was that $94 \%$ of the participants do not adopt any measure of hand hygiene for prevention of HAIs prior to patient contact despite $91 \%$ have the knowledge that hand hygiene should be performed before and after direct patient contact. The result of the present study showed that nurses had a good knowledge but poor practice in prevention of HAIs. A study in India reported less than desirable level of practice but a study in Nepal reported more among the health care personnel. ${ }^{11}$

\section{Conclusion}

The finding of this study revealed a good knowledge of infection prevention among the majority of participants with relatively minimal level of practice. However there is the need of regular training and performance feedback regarding hand hygiene and the hospital environment should be hand-hygiene friendly with easily accessible to sinks and other facilities. It is also necessary for an effective infection prevention team for updating of existing practices to reduce hospital acquired infections among staff nurses.

\section{References}

1. M.R. Meengs, B.K. Giles, C.D. Chisholm, W.H. Cordell, D.R. Nelson.Hand washing frequency in an emergency department.Journal of Emergency Nursing, 20 (1994), pp. 183-188

2. Lopez-Frias F, Castellanos-Cosano L, Martin-Gonzalez J, Llamas-Carreras J, Segura-Egea J. Clinical measurement of tooth wear: Tooth Wear Indices. J ClinExp Dent. 2012;4(1): e48-e53. DOI: 10.4317/jced.50592.

3. Lavatevestrasmanus. Handwashing Liaison Group.Annals of the Royal College of Surgeons of England, 82 (2000), pp. 133-136

4. Moro LM, Morsillo F, Tangenti M, Mongardi M, Pirazzini CM, Ragni P. Rates of surgical-site infection: An international comparison. Infection Control and Hospital Epidemiology. 2005;25(5):442-8. doi: https://doi.org/10.1086/ 502565. [PubMed]

5. Ishwari Sharama Paudel, Vivek Ghosh, Purushottam AdhikariKnowledge, Attitude and Practice of nursing students on Hospital Acquired Infections in Western region of Nepal, Journal of College of Medical Sciences-Nepal 2016;12(3):103-7.

6. H Sarani, A Balouchi, N Masinaeinezhad, E Ebrahimitabs; Knowledge, Attitude and Practice of Nurses about Standard Precautions for Hospital-Acquired Infection in Teaching Hospitals Affiliated to Zabol Universityof Medical Sciences: Global Journal of Health Science; Vol. 8, No. 3; 2016, 193-7

7. Amerioun, A., AA, K. Z., Tavakkoli, R., \& Zaboli, R. (2009). Supervisors' knowledge of hospital infections control in one of the medical sciences universities related hospitals. Journal Mil Med, 11, 11-12.

8. Saleh Moghadam, R. M. S. (2005). Knowledge and practice of nurses in relation with operating room pollution.J Knowl Manage., 39, 21-28.

9. Darawad, M. W. Al-Hussami, M. (2013). Jordanian nursing students' knowledge of, attitudes towards, and compliance with infection control precautions. Nurse education today, 33, 580-583. http://dx.doi.org/10.1016/j.nedt.2012.06.009).

10. P. Paudyal, P. Simkhada, J. Bruce.: Infection control knowledge, attitude, and practice among Nepalese health care workers. American Journal of Infection Control, 36 (2008), pp. 595-59

11. Yakob E, Lamaro T and Henok A. Knowledge, Attitude and Practice towards Infection Control Measures among Mizan-Aman General Hospital Workers, South West Ethiopia; J Community Med Health Educ 5:370. doi:10.4172/2161-0711.1000370

12. Suchitra, J. (2007). Impact of education on knowledge, attitudes and practices among various categories of health care workers on nosocomial infections. Indian journal of medical microbiology, $25,181$. http://dx.doi.org/10.4103/0255-0857.34757

13. Ribby K. Decreasing urinary tract infections through staff development, outcomes, and nursing process. Journal of Nursing Care Quality. 2005;21 (3):272-6.

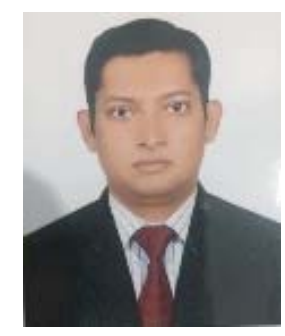

Dr. A S M Anwarul Kabir has passed MBBS from Holy Family Red Crescent Medical College in 2006. He is a student of FCPS part-2 in surgery under Bangladesh College of Physicians and surgeons. He is also achieving Masters of Public health under Bangladesh Open University. Now he is working as registrar in Surgery department at Holy Family Red Crescent Medical College and Hospital. 